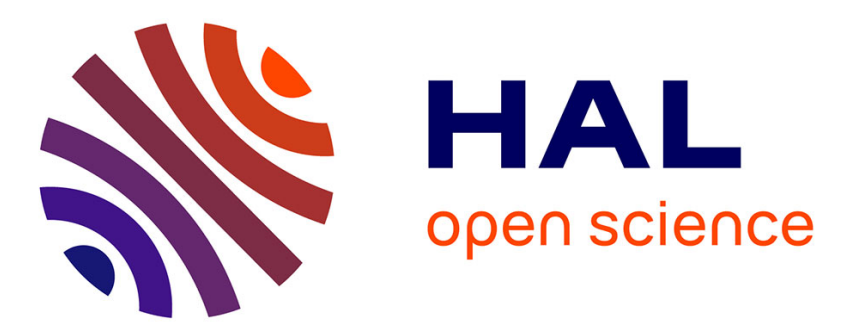

\title{
On finite-time stabilization of a class of nonlinear time-delay systems: Implicit Lyapunov-Razumikhin approach
}

Artem N Nekhoroshikh, Denis Efimov, Andrey Polyakov, Wilfrid Perruquetti, Igor B Furtat

\section{To cite this version:}

Artem N Nekhoroshikh, Denis Efimov, Andrey Polyakov, Wilfrid Perruquetti, Igor B Furtat. On finite-time stabilization of a class of nonlinear time-delay systems: Implicit Lyapunov-Razumikhin approach. CDC 2020 - 59th IEEE Conference on Decision and Control, Dec 2020, Jeju Island / Virtual, South Korea. hal-02942412

HAL Id: hal-02942412

https://hal.inria.fr/hal-02942412

Submitted on 17 Sep 2020

HAL is a multi-disciplinary open access archive for the deposit and dissemination of scientific research documents, whether they are published or not. The documents may come from teaching and research institutions in France or abroad, or from public or private research centers.
L'archive ouverte pluridisciplinaire HAL, est destinée au dépôt et à la diffusion de documents scientifiques de niveau recherche, publiés ou non, émanant des établissements d'enseignement et de recherche français ou étrangers, des laboratoires publics ou privés. 


\title{
On finite-time stabilization of a class of nonlinear time-delay systems: Implicit Lyapunov-Razumikhin approach
}

\author{
Artem N. Nekhoroshikh, Denis Efimov, Andrey Polyakov, Wilfrid Perruquetti, and Igor B. Furtat
}

\begin{abstract}
Theorems on Implicit Lyapunov-Razumikhin functions (ILRF) for asymptotic, exponential, finite-time and nearly fixed-time stability analysis of nonlinear time-delay systems are presented. Based on these results, finite-time stabilization of a special class of such systems is addressed. These systems are represented by a chain of integrators with a timedelay term multiplied by a function of instantaneous state vector. Possible explicit restriction on nonlinear time-delay terms is discussed. Simple procedure of control parameters calculation is given in terms of linear matrix inequalities (LMIs). Some aspects of digital implementations of the presented nonlinear control law are touched upon. Theoretical results are illustrated by numerical simulations.
\end{abstract}

\section{INTRODUCTION}

In many practical applications time response constraints are the main optimization criteria. For delay-free case there are plenty of techniques providing non-asymptotic (finitetime and fixed-time) rates of convergence [1], [2]. For example, by using theory of homogeneous dynamical systems, the rate of convergence depends on the degree of homogeneity of the system [2].

However, time-delay systems can be stabilized in finite time only under strong restrictions on the right-hand side of the system [3]. The most general sufficient conditions are given in [4]. On the other hand, in [3] the "necessary" condition for finite-time convergence is presented. It claims that the right-hand side of the system has to be equal to zero once the instantaneous state vector has reached the origin. For example, this condition will be satisfied if time-delay terms are multiplied by some functions depending on delayfree state vector or if the control contains distributed delays injection term.

There exist two main frameworks for stability analysis of time-delay systems: Lyapunov-Krasovskii approach and Lyapunov-Razumikhin method [5]. The former one is the natural extension of Lyapunov method for the case of timedelay systems, since delay-free state vector is replaced by the functional state vector. Therefore, this approach provides

A. N. Nekhoroshikh is with Faculty of Control Systems and Robotics, ITMO University, 197101, 49 Kronverkskiy av., Saint Petersburg, Russia annekhoroshikheitmo.ru

D. Efimov and A. Polyakov are with Valse team, Inria, Univ. Lille, CNRS, UMR 9189 - CRIStAL, F59000 Lille, France denis.efimov@inria.fr, andrey.polyakovdinria.fr

W. Perruquetti is with Faculty of Automation, Computer Engineering, Signal and Image Processing, Centrale Lille Institut, 59651, BP 48, Cité Scientifique, Villeneuve-d'Ascq, France wilfrid.perruquetti@centralelille.fr

I. B. Furtat is with IPME RAS, V.O., Bolshoj pr., 61 St. Petersburg, 199178, Russia cainenash@mail.ru qualitative as well as quantitative estimates on the system convergence. However, selection of Lyapunov-Krasovskii functional is not a trivial task. The latter method is based on a classical Lyapunov function, as the one employed for the delay-free case, and it imposes restrictions on time derivative of Lyapunov function only under auxiliary functional restrictions. Hence, it gives mainly a qualitative conclusion. Recently several extensions of Lyapunov-Razumikhin method have been proposed [6], allowing the rate of solution convergence to be estimated.

Nevertheless, the Lyapunov function (functional) methods being the most developed tool for analysis of non-asymptotic stability do not provide a constructive way to control synthesis. This drawback can be overcome by means of implicit formulation of Lyapunov-type theorems. The Implicit Lyapunov Function (ILF) method has been developed for delay-free systems in [7] as well as the Implicit Lyapunov-Krasovskii Functional (ILKF) approach has been proposed for timedelay systems in [8]. This methods allow one to check sufficient stability conditions and obtain control parameters by solving a system of LMIs.

Therefore, the goal of this work is to propose the implicit versions of Lyapunov-Razumikhin theorems on asymptotic and non-asymptotic stability developed in [6]. Differently from [8], the proposed approach allows one to use "simple" ILF (e.g. from [7]) rather than ILKF to design control laws. The obtained results are used to effectively solve a problem of finite-time stabilization of nonlinear time-delay systems. Moreover, some "mild sufficient" conditions on the right-hand side of the system are obtained. All stability conditions are represented by LMIs depending on two tuning parameters.

The outline of this work is as follows. The stability definitions of time-delay systems and explicit LyapunovRazumikhin theorems, guaranteeing each type of stability, are introduced in Section II. Implicit formulation of the theorems are given in Section III. The problem of finite-time stabilization of a special class of nonlinear time-delay systems is investigated in Section IV. The numerical simulation is presented in Section V.

Through the paper the following notations will be used:

- $\mathbb{R}$ is the field of real numbers, $\mathbb{R}_{>0}=\{x \in \mathbb{R}: x>0\}$ and $\mathbb{R}_{>0}=\mathbb{R}_{>0} \cup 0$;

- $\|\cdot\|$ is the Euclidean norm in $\mathbb{R}^{n},\|\cdot\|_{P}$ is the weighted Euclidean norm in $\mathbb{R}^{n}$ for a symmetric positive definite matrix $P \in \mathbb{R}^{n \times n}$;

- $\mathbb{C}_{h}$ is the space of continuous functions $[-h, 0] \rightarrow$ $\mathbb{R}^{n}$ with the norm $\|\cdot\|_{h}$ defined as follows $\|\Phi\|_{h}=$ 
$\max _{\tau \in[-h, 0]}\|\Phi(\tau)\|$ for $\Phi \in \mathbb{C}_{h}$

- a continuous function $\sigma: \mathbb{R}_{>0} \rightarrow \mathbb{R}_{>0}$ belongs to the class $\mathcal{K}$ if it is strictly increasing and $\sigma(0)=0$; if additionally it is unbounded then $\sigma$ belongs to $\mathcal{K}_{\infty}$;

- a series of integers $1,2, \ldots, n$ is denoted by $\overline{1, n}$;

- $\operatorname{diag}\left\{\lambda_{i}\right\}_{j=1}^{n}$ is the diagonal matrix with the elements $\lambda_{j} \in \mathbb{R}, j=\overline{1, n}$;

- if $P \in \mathbb{R}^{n \times n}$ is symmetric, then the inequalities $P>0$ $(P<0)$ and $P \geq 0(P \leq 0)$ mean that $P$ is positive (negative) definite and semidefinite, respectively;

- $\lambda_{\min }(P)$ and $\lambda_{\max }(P)$ are the minimal and maximal eigenvalues of a symmetric matrix $P \in \mathbb{R}^{n \times n}$, respectively;

- $I_{n} \in \mathbb{R}^{n \times n}$ is the identity matrix and $O_{n} \in \mathbb{R}^{n}$ is the zero column.

\section{Preliminaries}

Consider the time-delay system of the form [9]

$$
\begin{cases}\dot{x}(t)=\phi\left(x_{t}\right), & t \geq 0 \\ x(t)=\Phi(t), \Phi \in \mathbb{C}_{h}, & t \in[-h, 0]\end{cases}
$$

where $x(t) \in \mathbb{R}^{n}$ is the instantaneous state vector, $x_{t} \in$ $\mathbb{C}_{h}$ is the state of the delay system at time $t$, defined by $x_{t}(\tau)=x(t+\tau)$ with $\tau \in[-h, 0]$ for $h>0$ (time delay) and $\phi: \mathbb{C}_{h} \rightarrow \mathbb{R}^{n}$ is a continuous operator. Assume that the origin is an equilibrium point of the system (1), i.e. $\phi(0)=0$ for all $t \geq 0$. A solution of the system (1) with the initial function $\Phi \in \mathbb{C}_{h}$ is denoted by $x(t, \Phi)$.

\section{A. Stability definitions}

The definitions of Lyapunov, asymptotic, exponential, finite-time and nearly fixed-time stability [4] of the system (1) at the origin are given below.

Let $\mathcal{D}$ be a neighborhood of zero in $\mathbb{C}_{h}$.

Definition 1. At the origin the system (1) is said to be:

1) Lyapunov stable if there is $\varphi \in \mathcal{K}$ such that for any $\Phi \in$ $\mathcal{D}$, the solutions are defined and $\|x(t, \Phi)\| \leq \varphi\left(\|\Phi\|_{h}\right)$ for all $t \geq 0$;

2) asymptotically stable if it is Lyapunov stable and

$$
\lim _{t \rightarrow \infty}\|x(t, \Phi)\|=0 \quad \text { for any } \Phi \in \mathcal{D} ;
$$

3) exponentially stable if for any $\Phi \in \mathcal{D}$ there exist $\varphi^{\prime} \in \mathcal{K}$ and $\vartheta>0$ called the decay rate such that $\|x(t, \Phi)\| \leq$ $\varphi^{\prime}\left(\|\Phi\|_{h}\right) e^{-\vartheta t}$ for all $t \geq 0$

4) finite-time stable if it is Lyapunov stable and for any $\Phi \in \mathcal{D}$ there exists $0 \leq T_{\Phi}<\infty$ such that $x(t, \Phi)=0$ for all $t \geq T_{\Phi}$. The functional $T(\Phi)=\inf \left\{T_{\Phi} \geq 0\right.$ : $\left.x(t, \Phi)=0 \forall t \geq T_{\Phi}\right\}$ is called the settling time of the system (1);

5) nearly fixed-time stable if it is Lyapunov stable and for any $\varrho>0$ there exists $0<T(\varrho)<\infty$ called the transition time such that $\|x(t, \Phi)\| \leq \varrho$ for all $t \geq T(\varrho)$ and all $\Phi \in \mathcal{D}$.

If $\mathcal{D}=\mathbb{C}_{h}$, then the corresponding properties are called global Lyapunov/asymptotic/exponential/finite-time/nearly fixed-time stability.

\section{B. Razumikhin-type theorems}

Let us formulate a Lyapunov-Razumikhin theorem on asymptotic stability of time-delay systems [9] and its mild modifications [6] allowing one to estimate the exponential, finite-time and nearly fixed-time convergence rates. For brevity only the global stability will be considered in the sequel.

Denote values of $V$ at time instants $t$ and $t+\tau$ and derivative of $V$ with respect to time along the trajectories of the system (1) at time instant $t$ as follows: $V_{t}:=V\left(x_{t}(0)\right)$, $V_{t+\tau}:=V\left(x_{t}(\tau)\right), \dot{V}_{t}:=\left.\frac{\partial V(x)}{\partial x}\right|_{x=x_{t}(0)} \phi\left(x_{t}\right)$.

Theorem 1 (Theorem 4.2 [9]). Let there exists a locally Lipschitz continuous Lyapunov-Razumikhin function $V$ : $\mathbb{R}^{n} \rightarrow \mathbb{R}$ such that

1) for some $\sigma_{1}, \sigma_{2} \in \mathcal{K}_{\infty}$ and all $x \in \mathbb{R}^{n}$

$$
\sigma_{1}(\|x\|) \leq V(x) \leq \sigma_{2}(\|x\|) ;
$$

2a) for some $w, p \in \mathcal{K}$, with $p(s)>s$ for all $s>0$, and all $x_{t} \in \mathbb{C}_{h}$

$$
\max _{\tau \in[-h, 0]} V_{t+\tau} \leq p\left(V_{t}\right) \Rightarrow \dot{V}_{t} \leq-w\left(\left\|x_{t}(0)\right\|\right) .
$$

Then the system (1) is globally asymptotically stable at the origin.

Theorem 2 (Theorem 6 [6]). Let there exists a locally Lipschitz continuous Lyapunov-Razumikhin function $V$ : $\mathbb{R}^{n} \rightarrow \mathbb{R}$, satisfying condition 1) of Theorem 1, such that

$2 b)$ for some $\chi>1, \alpha>0$ and all $x_{t} \in \mathbb{C}_{h}$

$$
\max _{\tau \in[-h, 0]} V_{t+\tau} \leq \chi V_{t} \Rightarrow \dot{V}_{t} \leq-\alpha V_{t} .
$$

Then the origin of the system (1) is globally asymptotically stable with the decay rate of exponential convergence

$$
\vartheta=-\min \{\alpha, \ln (\chi) / h\} .
$$

Theorem 3 (Theorem 8 [6]). Let there exists a locally Lipschitz continuous Lyapunov-Razumikhin function $V$ : $\mathbb{R}^{n} \rightarrow \mathbb{R}$, satisfying condition 1) of Theorem 1, such that

2c) for some $\mu \in(-1,0), c>0, \alpha>0$ and all $x_{t} \in \mathbb{C}_{h}$

$$
\max _{\tau \in[-h, 0]} V_{t+\tau}^{-\mu} \leq V_{t}^{-\mu}+c h \Rightarrow \dot{V}_{t} \leq-\alpha V_{t}^{1+\mu} .
$$

Then the origin of the system (1) is globally finite-time stable with settling-time estimate

$$
T(\Phi)=\sigma_{2}^{-\mu}\left(\|\Phi\|_{h}\right) / \min \{-\alpha \mu, c\} .
$$

Theorem 4 (Theorem 9 [6]). Let there exists a locally Lipschitz continuous Lyapunov-Razumikhin function $V$ : $\mathbb{R}^{n} \rightarrow \mathbb{R}$, satisfying condition 1) of Theorem 1, such that

$2 d)$ for some $\nu>0, c>0, \alpha>0$ and all $x_{t} \in \mathbb{C}_{h}$

$$
\left(\max _{\tau \in[-h, 0]} V_{t+\tau}\right)^{-\nu}+c h \geq V_{t}^{-\nu} \Rightarrow \dot{V}_{t} \leq-\alpha V_{t}^{1+\nu} .
$$

Then the system (1) is globally nearly fixed-time stable at the origin with transition-time estimate to the ball $\mathcal{B}=\{x \in$ $\left.\mathbb{R}^{n}:\|x(t, \Phi)\| \leq \varrho\right\}$

$$
T(\varrho)=\sigma_{1}^{-\nu}(\varrho) / \min \{\alpha \nu, c\} .
$$




\section{IMPLICIT RAZUMIKHIN-TYPE THEOREMS}

In this subsection Theorems 1-4 are reformulated in the implicit way. Compared to explicit formulation, proposed approach is more constructive for control design purposes.

Before formulating the theorem concerning stability analysis of time-delay systems using ILRF, a special class of comparison functions is introduced in the following definition.

Definition 2 (Implicit comparison functions [8]). The function $q: \mathbb{R}_{>0}^{2} \rightarrow \mathbb{R}$ is said to be of the class $\mathcal{I} \mathcal{K}_{\infty}$ if:

1) $q$ is continuous on $\mathbb{R}_{>0}^{2}$;

2) for any $s \in \mathbb{R}_{>0}$ there exists $\sigma \in \mathbb{R}_{>0}$ such that $q(\sigma, s)=0$

3) for any fixed $s \in \mathbb{R}_{>0}$ the function $q(\cdot, s)$ is strictly decreasing on $\mathbb{R}_{>0}$;

4) for any fixed $\sigma \in \mathbb{R}_{>0}$ the function $q(\sigma, \cdot)$ is strictly increasing on $\mathbb{R}_{>0}$;

5) for all $(\sigma, s) \in \Gamma=\left\{(\sigma, s) \in \mathbb{R}_{>0}^{2}: q(\sigma, s)=0\right\}$, the following holds:

$$
\lim _{s \rightarrow 0^{+}} \sigma=0, \quad \lim _{\sigma \rightarrow 0^{+}} s=0, \quad \lim _{s \rightarrow \infty} \sigma=\infty .
$$

In other words, Definition 2 postulates that if $q \in \mathcal{I} \mathcal{K}_{\infty}$, then there exists a unique function $\sigma \in \mathcal{K}_{\infty}$ such that $q(\sigma(s), s)=0$ for all $s \in \mathbb{R}_{>0}$.

Denote values of $V$ and partial derivatives of function $Q(V, x)$ at time instant $t$ as follows: $V_{t}:=V(t), Q_{x}(t):=$ $\left.\frac{\partial Q(V, x)}{\partial x}\right|_{\substack{V=V_{t} \\ x=x_{t}(0)}}$ and $Q_{V}(t):=\left.\frac{\partial Q(V, x)}{\partial V}\right|_{\substack{V=V_{t} \\ x=x_{t}(0)}}$. The proofs of the following theorems are given in Appendix I.

Theorem 5 (Asymptotic stability). If there exists a continuous function $Q: \mathbb{R}_{>0} \times \mathbb{R}^{n} \rightarrow \mathbb{R}$ such that:

C1) $Q$ is continuously differentiable outside the origin;

C2) for any $x \in \mathbb{R}^{n} \backslash\{0\}$ there exists $V \in \mathbb{R}_{>0}$ such that $Q(V, x)=0$;

C3) there exist $q_{1}, q_{2} \in \mathcal{I} \mathcal{K}_{\infty}$ such that for all $V \in \mathbb{R}_{>0}$ and $x \in \mathbb{R}^{n} \backslash\{0\}$ :

$$
q_{1}(V,\|x\|) \leq Q(V, x) \leq q_{2}(V,\|x\|)
$$

C4) $\frac{\partial Q(V, x)}{\partial V}<0$ for all $V \in \mathbb{R}_{>0}$ and $x \in \mathbb{R}^{n} \backslash\{0\}$;

C5a) for all $x_{t} \in \mathbb{C}_{h}$ and $\left(V_{t}, x_{t}(0)\right) \in \Omega=\left\{\left(V_{t}, x_{t}(0)\right) \in\right.$ $\left.\mathbb{R}_{>0} \times \mathbb{R}^{n}: Q\left(V_{t}, x_{t}(0)\right)=0\right\}$.

$$
\begin{gathered}
\max _{\tau \in[-h, 0]} Q\left(p\left(V_{t}\right), x_{t}(\tau)\right) \leq 0 \Rightarrow \\
Q_{x}(t) \phi\left(x_{t}\right) \leq w\left(\left\|x_{t}(0)\right\|\right) Q_{V}(t),
\end{gathered}
$$

where $p, w \in \mathcal{K}$ and $p(V)>V$ for all $V \in \mathbb{R}_{>0}$. Then the origin of the system (1) is globally asymptotically stable.

Theorem 6 (Exponential stability). If there exists a continuous function $Q: \mathbb{R}_{>0} \times \mathbb{R}^{n} \rightarrow \mathbb{R}$ such that conditions C1-C4 of Theorem 5 are satisfied and the condition:

C5b) for all $x_{t} \in \mathbb{C}_{h}$ and $\left(V_{t}, x_{t}(0)\right) \in \Omega$ there exist $\chi>1, \alpha>0$ such that:

$$
\max _{\tau \in[-h, 0]} Q\left(\chi V_{t}, x_{t}(\tau)\right) \leq 0 \Rightarrow Q_{x}(t) \phi\left(x_{t}\right) \leq \alpha V_{t} Q_{V}(t) .
$$

Then the origin of the system (1) is globally asymptotically stable with the decay rate of exponential convergence (2).

Theorem 7 (Finite-time stability). If there exists a continuous function $Q: \mathbb{R}_{>0} \times \mathbb{R}^{n} \rightarrow \mathbb{R}$ such that conditions C1-C4 of Theorem 5 are satisfied and the condition:

C5c) for all $x_{t} \in \mathbb{C}_{h}$ and $\left(V_{t}, x_{t}(0)\right) \in \Omega$ there exist $\mu \in(-1,0), c>0$ and $\alpha>0$ such that:

$$
\begin{gathered}
\max _{\tau \in[-h, 0]} Q\left(\left(V_{t}^{-\mu}+c h\right)^{-1 / \mu}, x_{t}(\tau)\right) \leq 0 \Rightarrow \\
Q_{x}(t) \phi\left(x_{t}\right) \leq \alpha V_{t}^{1+\mu} Q_{V}(t) .
\end{gathered}
$$

Then the origin of the system (1) is globally finite-time stable with settling-time estimate (3), where $\sigma_{2} \in \mathcal{K}_{\infty}$ such that $q_{2}\left(\sigma_{2}(s), s\right)=0$ for all $s \in \mathbb{R}_{>0}$.

Theorem 8 (Nearly fixed-time stability). If there exists a continuous function $Q: \mathbb{R}_{>0} \times \mathbb{R}^{n} \rightarrow \mathbb{R}$ such that conditions C1-C4 of Theorem 5 are satisfied and the condition:

C5d) for all $x_{t} \in \mathbb{C}_{h}$ and $\left(V_{t}, x_{t}(0)\right) \in \Omega$ there exist $\nu>0, c>0$ and $\alpha>0$ such that:

$$
\begin{gathered}
\max _{\tau \in[-h, 0]} Q\left(\left(V_{t}^{-\nu}-c h\right)^{-1 / \nu}, x_{t}(\tau)\right) \leq 0 \Rightarrow \\
Q_{x}(t) \phi\left(x_{t}\right) \leq \alpha V_{t}^{1+\nu} Q_{V}(t) .
\end{gathered}
$$

Then the system (1) is globally nearly fixed-time stable at the origin with transition-time estimate (4), where $\sigma_{1} \in \mathcal{K}_{\infty}$ such that $q_{1}\left(\sigma_{1}(s), s\right)=0$ for all $s \in \mathbb{R}_{>0}$.

\section{FInITE-TIME STABILIZATION OF TIME-DELAY SYSTEMS}

\section{A. Problem statement}

Let us consider a nonlinear time-delay system of the form:

$$
\left\{\begin{array}{l}
\dot{x}(t)=A x(t)+b u(t)+F(x(t)) x(t-h), t \geq 0 \\
x(t)=\Phi(t), \quad \Phi \in \mathbb{C}_{h}, \quad t \in[-h, 0]
\end{array}\right.
$$

where $x(t) \in \mathbb{R}^{n}$ is the instantaneous state vector, $u(t) \in \mathbb{R}$ is the control input, $h>0$ is the known time delay; the system matrix $A$, the vector $b \in \mathbb{R}^{n}$ and the function gain $F: \mathbb{R}^{n} \rightarrow \mathbb{R}^{n \times n}$ are of the form:

$$
\begin{gathered}
A=\left[\begin{array}{cc}
O_{n-1} & I_{n-1} \\
0 & O_{n-1}^{T}
\end{array}\right], b=\left[\begin{array}{c}
O_{n-1} \\
1
\end{array}\right], \\
F(x(t))=\left[\begin{array}{cc}
O_{n-1} & \operatorname{diag}\left\{f_{j}(x(t))\right\}_{j=1}^{n-1} \\
0 & O_{n-1}^{T}
\end{array}\right] .
\end{gathered}
$$

Functions $f_{j}(x(t)), j=\overline{1, n-1}$ are continuous and $f_{j}(0)=$ 0 . The function $\Phi \in \mathbb{C}_{h}$ represents initial conditions.

The problem is to stabilize the origin of the system (5) in finite time. Let us note that the well-known obstruction to finite-time stabilization of time delay systems (see [3], [4]) is overcome since the right-hand side of the system is zero once the instantaneous state vector has reached the origin (for $u=0$ ). 


\section{B. Control design}

Introduce the Implicit Lyapunov-Razumikhin function candidate [7]

$$
Q(V, x):=x^{\top} D\left(V^{-1}\right) P D\left(V^{-1}\right) x-1,
$$

where $V \in \mathbb{R}_{>0}, x \in \mathbb{R}^{n}, 0<P=P^{\top} \in \mathbb{R}^{n \times n}, D(\lambda):=$ $\operatorname{diag}\left\{\lambda^{r_{i}}\right\}_{i=1}^{n}$ is a diagonal matrix with $r=\left[r_{1}, \ldots, r_{n}\right]^{\top} \in$ $\mathbb{R}_{>0}^{n}$ and

$$
r_{i}=1-(n-i) \mu, \quad-1<\mu<0 .
$$

Denote $H:=\operatorname{diag}\left\{-r_{i}\right\}_{i=1}^{n}$.

Theorem 9 (Finite-time stabilization). Let there exists $\mu \in(-1,0)$ such that the following conditions hold:

1) the functions $f_{j}(x), j=\overline{1, n-1}$ satisfy the inequalities

$$
\left|f_{j}(x)\right| \leq \delta_{0}\left(\frac{V^{-\mu}}{V^{-\mu}+c h}\right)^{-r_{j+1} / \mu}
$$

for some $c, \delta_{0}>0$ and all $V \in \mathbb{R}_{\geq 0}, x \in \mathbb{R}^{n}$ such that $Q(V, x)=0$.

2) there exist $\alpha, \gamma, \rho, \eta>0$ such that LMIs

$$
\begin{gathered}
-\gamma X \leq X H+H X<0 \\
A X+b y^{\top}+X A^{\top}+y b^{\top}+\left(2 \alpha \gamma+\rho^{-1}\right) X \leq 0 \\
\delta_{0}^{2} \eta \rho /(\alpha \gamma) I_{n} \leq X \leq \eta I_{n}
\end{gathered}
$$

are feasible for some $0<X=X^{\top} \in \mathbb{R}^{n \times n}$ and $y \in \mathbb{R}^{n}$;

$3)$ the control law $u$ has the form

$$
u(V, x)=V^{1+\mu} k^{\top} D\left(V^{-1}\right) x,
$$

where $k^{\top}:=y^{\top} X^{-1}, V \in \mathbb{R}_{>0}$ is such that $Q(V, x)=0$ and $Q$ is defined by (6) with $P=X^{-1}$;

Then the closed-loop system (5), (9) is globally finite-time stable with settling-time estimate

$$
T(\Phi)=\frac{\left[\max \left\{\kappa^{1 /\left(2 r_{1}\right)}\left(\|\Phi\|_{h}\right), \kappa^{1 / 2}\left(\|\Phi\|_{h}\right)\right\}\right]^{-\mu}}{\min \{-\alpha \mu, c\}}
$$

where $\kappa\left(\|\Phi\|_{h}\right):=\alpha \gamma /\left(\delta_{0}^{2} \eta \rho\right)\|\Phi\|_{h}^{2}$.

Let us make some comments about the presented control scheme:

Firstly, the practical implementation of the control law (9) requires to find the solution $V(x)$ of the equation $Q(V, x)=$ 0 . Since $Q(V, x)=0$ is a monotonically decreasing function for any fixed $x \in \mathbb{R}^{n} \backslash\{0\}$, then the value $V(x)$ can be found numerically on-line using the current value of the state vector. To this end, one can use, for example, Algorithm 10 from [8] based on the bisection method. This algorithm allows to stabilize the system (5) in the vicinity of the origin, which is defined by $V_{\min }$, the smallest possible value of $V$.

Secondly, the closed-loop system (5), (9) satisfies the necessary condition for a finite-time convergence, i.e. $\dot{x}(t)=$ 0 when $x(t)=0$ [3], [4]. Indeed, $x(t)=0$ implies that $V(t)=0$, then it follows from (7) and (9) that $f_{j}(0)=$ $0, j=\overline{1, n-1}$ and $u(0,0)=0$, respectively. Therefore, the necessary condition is embedded in the "sufficient" one (7).
However, restrictions (7) on functions $f_{j}(x)$ are given in the form dependent on ILRF $V$, then in order to suggest an interpretation of (7), the next proposition provide a possible explicit form of functions $f_{j}(x)$.

Proposition 10. Condition (7) of Theorem 9 is satisfied if functions $f_{j}(x)$ are defined as follows:

$$
f_{j}(x):=\delta_{j}\left(\frac{\|x\|_{r}^{-\mu}}{\|x\|_{r}^{-\mu}+\beta_{j}}\right)^{-r_{j+1} / \mu}
$$

where $\|x\|_{r}$ is a homogeneous norm, defined as follows

$$
\|x\|_{r}:=\left(\sum_{i=1}^{n}\left|x_{i}\right|^{r_{1} / r_{i}}\right)^{1 / r_{1}}
$$

and

$$
0 \leq\left|\delta_{j}\right| \leq \delta_{0}, \quad \beta_{j} \geq \beta_{0}:=\operatorname{ch}\left(\sum_{i=1}^{n} \eta^{r_{1} /\left(2 r_{i}\right)}\right)^{-\mu / r_{1}}>0
$$

Proof. Clearly, it is sufficient to prove that for all $V \in \mathbb{R}_{\geq 0}$, $x \in \mathbb{R}^{n}$ such that $Q(V, x)=0$ the following relation holds

$$
\|x\|_{r} \leq V\left(\sum_{i=1}^{n} \eta^{r_{1} /\left(2 r_{i}\right)}\right)^{1 / r_{1}}
$$

Taking into account that the homogeneous norm is a $r$-homogeneous function of degree one, i.e. $\|x\|_{r}=$ $V\left\|D\left(V^{-1}\right) x\right\|_{r}$, then it yields

$$
\left(\sum_{i=1}^{n}\left|V^{-r_{i}} x_{i}\right|^{r_{1} / r_{i}}\right)^{1 / r_{1}} \leq\left(\sum_{i=1}^{n} \eta^{r_{1} /\left(2 r_{i}\right)}\right)^{1 / r_{1}} .
$$

Since for all $(V, x) \in \Omega$ we have $\left|V^{-r_{i}} x_{i}\right| \leq \lambda_{\min }^{-1 / 2}(P)$ and $\lambda_{\min }^{-1}(P) \leq \eta$ due to (8c), then the condition (7) is satisfied for functions (11).

Thirdly, by means of (10) a settling-time estimate could be obtained.

Fourthly, the number of the design parameters in Theorem 9 can be significantly reduced, if one let $\rho=\alpha=1$ and $\gamma=2(1-n \mu)$. Moreover, parameter $\eta$ can be chosen, for example, as the solution of the equation

$$
\left(\sum_{i=1}^{n} \eta^{r_{1} /\left(2 r_{i}\right)}\right)^{-\mu / r_{1}}=\beta_{0} /(c h)
$$

Fifthly, the parameter $c$ determines the rate of convergence in the system and affects on tuning parameter $\eta$ from LMIs (8). Thus, by restricting the converge rate with $c=1 / h$ it is possible to obtain delay-independent LMIs to check.

Summarizing all remarks, we can formulate the following corollary.

Corollary 11. Let there exist $\mu \in(-1,0), c>0$ such that:

1) the functions $f_{j}(x)$ are defined by (11) for some given $\delta_{0}, \beta_{0}>0$; 
2) there exists $\eta>0$ satisfying equation (12) such that LMIs

$$
\begin{gathered}
-2(1-n \mu) X \leq X H+H X<0 \\
A X+b y^{\top}+X A^{\top}+y b^{\top}+[(4-4 n \mu)+1] X \leq 0 \\
\delta_{0}^{2} \eta /(2-2 n \mu) I_{n} \leq X \leq \eta I_{n}
\end{gathered}
$$

are feasible for some $0<X=X^{\top} \in \mathbb{R}^{n \times n}$ and $y \in \mathbb{R}^{n}$.

Then the closed-loop system (5), (9) is globally finite-time stable with settling-time estimate

$$
T(\Phi)=\frac{\left[\max \left\{\tilde{\kappa}^{1 /\left(2 r_{1}\right)}\left(\|\Phi\|_{h}\right), \tilde{\kappa}^{1 / 2}\left(\|\Phi\|_{h}\right)\right\}\right]^{-\mu}}{\min \{-\mu, c\}},
$$

where $\tilde{\kappa}\left(\|\Phi\|_{h}\right):=2(1-n \mu) /\left(\delta_{0}^{2} \eta\right)\|\Phi\|_{h}^{2}$.

Therefore, the proposed control algorithm allows one to choose $\mu \in(-1,0)$ and $c>0$ such that the closed-loop system (5), (9) with functions gains (11) is stabilized in finite time. Obviously, the system of LMIs (13) is feasible for sufficiently small $\delta_{0}$ due to the controllability (in the classical linear sense) of the pair $(A, b)$. Parameter $\eta$ can be chosen arbitrarily large for given $\beta_{0}$ and $h$, since $c$ is the tuning parameter.

\section{Simulation}

Consider the system (5) for $n=3$, with functions $f_{j}(x)$ described by (11). Assume that $\delta_{0}=0.01$ and $\beta_{0}=1$. From (14) it follows that both $\mu$ and $c$ define the settling time $T(\Phi)$. However, let us fix $c=1 / h$ in order to obtain delayindependent LMIs. Therefore, by solving equation (12) and choosing minimal $\mu$, for which LMIs (13) are feasible, we obtain $\eta=0.2337, \mu=-0.75$ and

$K=\left[\begin{array}{c}-1822.20 \\ -371.61 \\ -24.21\end{array}\right], P=\left[\begin{array}{ccc}215824.29 & 29817.52 & 1029.93 \\ 29817.52 & 5130.00 & 209.91 \\ 1029.93 & 209.91 & 14.20\end{array}\right]$

Let us compare performance of the proposed control law (9) with corresponding linear one $(\mu=0)$ by numerical simulations with respect to different initial conditions and time delays. During the simulation, initial conditions $\Phi$ are assumed to be constant, i.e. $\Phi(\tau)=\|\Phi\|_{h}$ for all $\tau \in[-h, 0]$, and parameters $\delta_{j}, \beta_{j}, j=1,2$ are chosen as follows:

$$
\delta_{1}=-0.0078, \delta_{2}=0.0074, \beta_{1}=1.3175, \beta_{2}=1.0290 \text {. }
$$

The following numerical simulation has been done in MATLAB Simulink using the Euler method with a fixed step size $\Delta t=10^{-4}$. The control has been applied using Algorithm 10 [8] with $V_{\min }=10^{-3}$.

\section{A. Different initial conditions}

The norm of the trajectories $\|x(t)\|$ of the system (5) with $h=1$ second are depicted on Fig. 1 in the logarithmic scale for different initial conditions. Solid and dashed lines correspond to nonlinear (NSFB) and linear state feedback (LSFB). Clearly, the proposed control law preserves finitetime stability for different initial conditions. Moreover, the settling time is proportional to the norm of initial conditions $\|\Phi\|_{h}$, that agrees with (14).

\section{B. Different time delays}

The norm of the trajectories $\|x(t)\|$ of the system (5) are presented on Fig. 2 in the logarithmic scale for different values of time delay. Solid and dashed lines correspond to nonlinear (NSFB) and linear state feedback (LSFB), meanwhile, red, black and blue ones correspond to time delay $h=2, h=1$ and $h=0.5$ seconds, respectively. Obviously, the proposed control law preserves finite-time stability for different values of time delays.

\section{CONCLUSION}

The paper develops the Implicit Lyapunov-Razumikhin Function method for asymptotic, exponential, finite-time and nearly fixed-time stability analysis of nonlinear timedelay systems. The problem of finite-time stabilization of a special class of such systems has been solved by means of the proposed method. The implicit approach provides an effective way of control parameters computation, having LMI representation. For some particular cases LMIs have been shown to depend only on two tuning parameters. The numerical simulation for different initial conditions and time delays has confirmed obtained theoretical results. Application of the developed method to more sophisticated time-delay systems is a possible direction of future research.

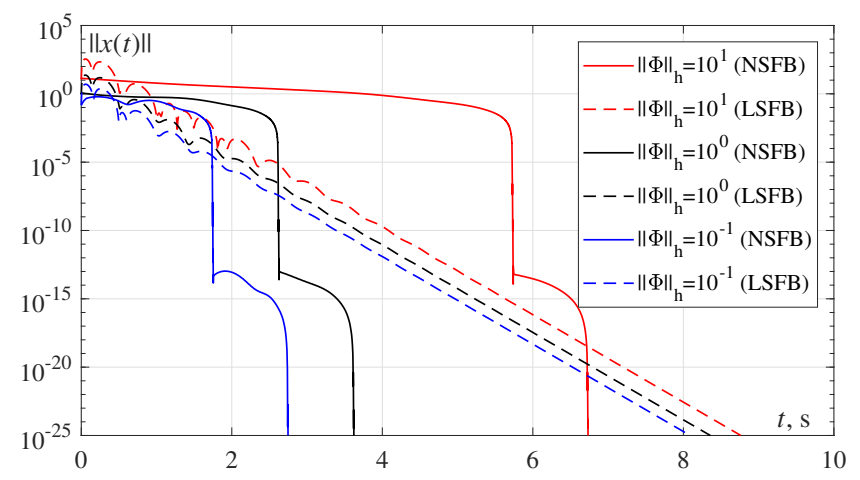

Fig. 1. Comparison of nonlinear (NSFB) and linear state feedback (LSFB) for different initial conditions

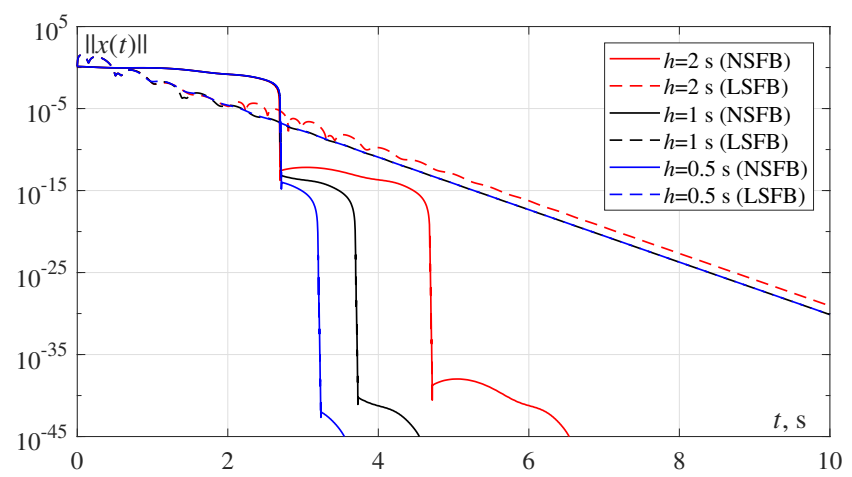

Fig. 2. Comparison of nonlinear (NSFB) and linear state feedback (LSFB) for different time delays 


\section{ACKNOWLEDGMENTS}

This work has been partially supported by ANR Project Finite4SoS (ANR 15-CE23-0007), by the Government of Russian Federation (Grant 08-08), by the Ministry of Science and Higher Education of Russian Federation, passport of goszadanie no. 2019-0898 and by the RFBR research project no. 19-08-00246.

\section{REFERENCES}

[1] Y. Orlov. Finite time stability and robust control synthesis of uncertain switched systems. SIAM Journal of Control and Optimization, vol. 43(4), pp. 1253-1271, 2005.

[2] E. Bernuau, D. Efimov, W. Perruquetti, and A. Polyakov. On homogeneity and its application in sliding mode. International Journal of Franklin Institute, vol. 351, no. 4, pp. 1866-1901, 2014.

[3] D. Efimov, A. Polyakov, E. Fridman, W. Perruquetti, J.-P. Richard. Comments on Finite-time Stability of Time-Delay Systems. Automatica, Elsevier, 50 (7), pp.1944-1947, 2014.

[4] E. Moulay, M. Dambrine, N. Yeganefar, and W. Perruquetti. Finitetime stability and stabilization of time-delay systems. Systems \& Control Letters, 57 (7), pp.561-566, 2008.

[5] K. Gu, V. L. Kharitonov, and J. Chen. Stability of time-delay systems. Birkhauser, 2003.

[6] D. Efimov and A. Aleksandrov. On estimation of rates of convergence in Lyapunov-Razumikhin approach. Automatica, 2020.

[7] A. Polyakov, D. Efimov, and W. Perruquetti. Finite-time stabilization using implicit lyapunov function technique. In 9th Symposium on Nonlinear Control Systems, pp. 140-145, 2013.

[8] A. Polyakov, D. Efimov, W. Perruquetti and J.P. Richard. Implicit Lyapunov-Krasovski Functionals For Stability Analysis and Control Design of Time-Delay Systems. IEEE Transactions on Automatic Control. 2015

[9] J. Hale. Theory of Functional Differential Equations. Springer-Verlag, 1977.

[10] R. Courant and F. John. Introduction to calculus and analysis (Vol. II/1). New York: Springer, 2000.

\section{APPENDIX I}

\section{PROOF OF THEOREMS 5-8}

In order to prove Theorems 5-8, let us show that there exists a function $V: \mathbb{R}^{n} \rightarrow \mathbb{R}_{\geq 0}$ that satisfies conditions of Theorems 1-4. It was proven in [7] that conditions C1)-C4) from Theorem 5 imply condition 1) from Theorem 1.

On the other hand, conditions 2a)-2b) and C5a)-C5b) are equivalent. Indeed, for any $\tau \in[-h, 0]$ take $\left(V_{t+\tau}, x_{t}(\tau)\right) \in$ $\Omega$. Since the function $Q(s, x)$ is monotonically decreasing for any fixed $x \in \mathbb{R}^{n} \backslash\{0\}$ (C4), we have the following equivalence for any $s \in \mathbb{R}_{>0}$ :

$$
\max _{\tau \in[-h, 0]} Q\left(s, x_{t}(\tau)\right) \leq 0 \Leftrightarrow \max _{\tau \in[-h, 0]} V_{t+\tau} \leq s .
$$

Taking this into account and that the classical implicit function theorem [10] for Euclidean spaces implies that $\dot{V}_{t}=-Q_{x}(t) f\left(x_{t}\right) / Q_{V}(t)$, conditions C5a)-C5d) can be rewritten respectively as

$$
\begin{aligned}
\max _{\tau \in[-h, 0]} V_{t+\tau} \leq p\left(V_{t}\right) & \Rightarrow \dot{V}_{t} \leq-w\left(\left\|x_{t}(0)\right\|\right) \\
\max _{\tau \in[-h, 0]} V_{t+\tau} \leq \chi V_{t} & \Rightarrow \dot{V}_{t} \leq-\alpha V_{t} \\
\max _{\tau \in[-h, 0]} V_{t+\tau} \leq\left(V_{t}^{-\mu}+c h\right)^{-1 / \mu} & \Rightarrow \dot{V}_{t} \leq-\alpha V_{t}^{1+\mu} \\
\max _{\tau \in[-h, 0]} V_{t+\tau} \leq\left(V_{t}^{-\nu}-c h\right)^{-1 / \nu} & \Rightarrow \dot{V}_{t} \leq-\alpha V_{t}^{1+\nu} .
\end{aligned}
$$

Since $-\mu>0$ and $-\nu<0$, these conditions are equivalent to the corresponding ones from $2 \mathrm{a}$ )-2d).
APPENDIX II

\section{PROOF OF THEOREM 9}

It can be shown that the following inequalities

$$
\begin{aligned}
q_{1}(V,\|x\|) & =\frac{\lambda_{\min }(P)\|x\|^{2}}{\max \left\{V^{2-2(n-1) \mu}, V^{2}\right\}}-1 \leq Q(V, x) \\
& \leq \frac{\lambda_{\max }(P)\|x\|^{2}}{\min \left\{V^{2-2(n-1) \mu}, V^{2}\right\}}-1=q_{2}(V,\|x\|)
\end{aligned}
$$

hold for all $V \in \mathbb{R}_{>0}$ and $x \in \mathbb{R}^{n}$. Therefore, the function (6) satisfies conditions C1)-C3) of Theorem 5.

The condition $\mathrm{C} 4$ ) of Theorem 5 also holds, since from (8a) it follows

$$
0>\frac{\partial Q(V, x)}{\partial V}=V^{-1} x^{\top} D\left(V^{-1}\right)[H P+P H] D\left(V^{-1}\right) x .
$$

Taking into account that $D\left(V^{-1}\right) A=V^{\mu} A D\left(V^{-1}\right)$, $D\left(V^{-1}\right) F(x)=V^{\mu} F(x) D\left(V^{-1}\right)$ and $D\left(V^{-1}\right) b u=$ $V^{\mu} b k^{\top} D\left(V^{-1}\right) x$, we obtain:

$$
\begin{gathered}
Q_{x}(t) f\left(x_{t}\right)=2 V_{t}^{\mu} x_{t}(0)^{\top} D\left(V_{t}^{-1}\right) P \\
\times\left[\left(A+b k^{\top}\right) D\left(V_{t}^{-1}\right) x_{t}(0)+F\left(x_{t}(0)\right) D\left(V_{t}^{-1}\right) x_{t}(-h)\right] .
\end{gathered}
$$

Denote $d\left(x_{t}\right):=F\left(x_{t}(0)\right) D\left(V_{t}^{-1}\right) x_{t}(-h)$ and $z\left(x_{t}\right):=$ $\left[x_{t}(0) ; d\left(x_{t}\right)\right]$. Then adding and subtracting $\rho V_{t}^{\mu}\left\|d\left(x_{t}\right)\right\|_{P}^{2}$ and $2 \alpha \gamma V_{t}^{\mu}$, we get:

$Q_{x}(t) f\left(x_{t}\right)=-2 \alpha \gamma V_{t}^{\mu}+V_{t}^{\mu} z^{\top}\left(x_{t}\right) \Psi z\left(x_{t}\right)+\rho V_{t}^{\mu}\left\|d\left(x_{t}\right)\right\|_{P}^{2}$,

where the matrix

$$
\Psi:=\left[\begin{array}{cc}
P\left(A+b k^{\top}\right)+\left(A+b k^{\top}\right)^{\top} P+2 \alpha \gamma P & P \\
P & -\rho P
\end{array}\right]
$$

is negative semidefinite due to (8b) and the Schur complement. Using (8c) and (7), disturbance term $\rho\left\|d\left(x_{t}\right)\right\|_{P}^{2}$ could be bounded as:

$$
\begin{gathered}
\rho\left\|d\left(x_{t}\right)\right\|_{P}^{2} \leq \frac{\alpha \gamma}{\delta_{0}^{2} \eta} \sum_{j=1}^{n-1}\left|f_{j}\left(x_{t}(0)\right) V_{t}^{-r_{j+1}} x_{t, j+1}(-h)\right|^{2} \\
\leq \frac{\alpha \gamma}{\eta} \sum_{j=1}^{n-1}\left(V_{t}^{-\mu}+c h\right)^{2 r_{j+1} / \mu}\left|x_{t, j+1}(-h)\right|^{2} .
\end{gathered}
$$

Therefore, taking into account (8c), one could deduce that:

$Q_{x}(t) f\left(x_{t}\right) \leq-\alpha \gamma V_{t}^{\mu}\left[2-\left\|D\left(\left(V_{t}^{-\mu}+c h\right)^{1 / \mu}\right) x_{t}(-h)\right\|_{P}^{2}\right]$.

Finally, applying Theorem 7, one can see that for all $x_{t} \in \mathbb{C}_{h}$ and $\left(V_{t}, x_{t}(0)\right) \in \Omega$ such that

$$
\begin{gathered}
Q\left(\left(V_{t}^{-\mu}+c h\right)^{-1 / \mu}, x_{t}(-h)\right)<0 \Leftrightarrow \\
\left\|D\left(\left(V_{t}^{-\mu}+c h\right)^{1 / \mu}\right) x_{t}(-h)\right\|_{P}^{2}<1
\end{gathered}
$$

the following estimation holds:

$$
Q_{x}(t) f\left(x_{t}\right) \leq-\alpha \gamma V_{t}^{\mu} \leq \alpha V_{t}^{1+\mu} Q_{V}(t) .
$$

Hence, condition $\mathrm{C} 5 \mathrm{c}$ ) from Theorem 7 holds.

From Theorem 3 it follows that system (1) will be stabilized at the origin in finite time $T(\Phi)$ such that $\sigma_{2}^{-\mu}\left(\|\Phi\|_{h}\right)=\min \{-\alpha \mu, c\} T(\Phi)$. Since $\sigma_{2}(s)$ is implicitly defined by function $q_{2}\left(\sigma_{2}(s), s\right)=0$ and $\lambda_{\max }(P) \leq \alpha \gamma /\left(\delta_{0}^{2} \eta \rho\right)$ due to (8c), then $\sigma_{2}(s) \leq$ $\max \left\{\kappa^{1 /\left(2 r_{1}\right)}(s), \kappa^{1 / 2}(s)\right\}$. Therefore, from (3) we get the settling-time estimate (10). 